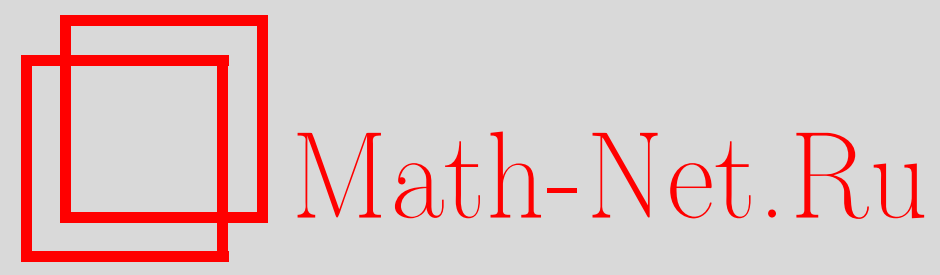

Н. Г. Докучаев, Локальное время пребывания диффузионных и вырождающихся процессов на подвижной поверхности, Теория вероятн. и ее примен., 1998, том 43, выпуск $2,226-247$

DOI: https://doi.org/10.4213/tvp1463

Использование Общероссийского математического портала Math-Net.Ru подразумевает, что вы прочитали и согласны с пользовательским соглашением http://www . mathnet.ru/rus/agreement

Параметры загрузки:

IP: 54.237 .206 .68

26 апреля 2023 г., $07: 26: 40$

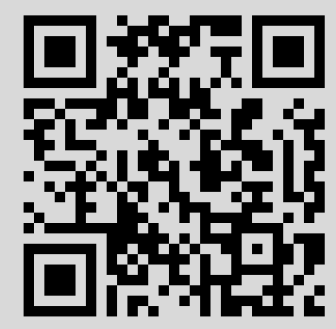


(C) $1998 \mathrm{r}$.

\author{
ДОКУЧАЕВ Н. Г.*
}

\title{
ЛОКАЛЬНОЕ ВРЕМЯ ПРЕБЫВАНИЯ ДИФФУЗИОННЫХ И ВЫРОЖДАЮЩИХСЯ ПРОЩЕССОВ НА ПОДВИЖНОЙ ПОВЕРХНОСТИ ${ }^{1)}$
}

\begin{abstract}
Для многомерных процессов Ито установлено существование локального времени пребывания на поверхности и выведены уравнения для вероятностных распределений. Получена явная формула для локального времени типа формулы Танаки. Установлена непрерывность локального времени. Исследованы предельные свойства локального времени при вырождении диффузии.
\end{abstract}

Ключевые слова и Фразы: локальное время, диффузионные процессы, вырождение диффузии.

Введение. Вопросам, связанным с вероятностным распределением функционалов от локального времени, посвящена обширная литература (см. обзоры [1]-[3] и библиографию там же). Наиболее подробно изучено локальное время скалярного броуновского движения. Представляется естественным иметь описание распределения локального времени для более общих процессов, чем броуновское движение (например, для диффузионных процессов с зависящим от управления сносом, чтобы включить в стохастическую теорию оптимального управления новые задачи, в которых требуется минимизировать или максимизировать локальное время пребывания в множествах нулевой меры). Существование локального времени пребывания на гладких гиперповерхностях для общих многомерных семимартингалов было установлено в [4], при этом распределение локального времени не изучалось. С использованием аппарата уравнений Колмогорова ниже получено описание распределения локального времени пребывания для общего многомерного марковского диффузионного процесса на изменяющейся во времени «кусочногладкой» или даже фрактальной гиперповерхности (пример 4.1); выведены специальные аналоги уравнений Колмогорова; установлена разрешимость этих уравнений, а также сходимость случайных величин, предел

*С.-Петербургский государственный университет, НИИ математики и механики, 198904 С.-Петербург, Россия.

1) Работа выполнена при поддержке Российского фонда фундаментальных исследований, грант № 96-01-00408. 
которых обычно называют локальным временем. Найдено явное выражение локального времени через стохастический интеграл, те. получен аналог формулы Танаки. Изучены предельные свойства локалького времени при исчезновении диффузии, когда уравнение Ито переходит в обыкновенное дифференциальное уравнение.

1. Постановка задачи. На вероятностном пространстве $(\Omega, \mathscr{F}, \mathbf{P})$ рассмотрим $n$-мерный винеровский процесс $w(t)$ с независимыми компонентами такой, что $w(0)=0$. Рассмотрим случайный $n$-вектор $a$, не зависящий от $w(t)$. Пусть случайный процесс $y(t)$ рәзмерности $n$ является сильным решением стохастического уравнения Ито

$$
\begin{gathered}
d y(t)=f(y(t), t) d t+\dot{\beta}(y(t), t) d w(t), \\
y(0)=a .
\end{gathered}
$$

Функции $f(x, t): \mathbf{R}^{n} \times \mathbf{R} \longrightarrow \mathbf{R}^{n}, \boldsymbol{\beta}(x, t): \mathbf{R}^{n} \times \mathbf{R} \longrightarrow \mathbf{R}^{n \times n}$ ограничены и измеримы. Полагаем, что функция $\beta(x, t)$ непрерывна, производная $\partial \beta(x, t) / \partial x$ ограничена, $b(x, t)=\frac{1}{2} \beta(x, t) \beta(x, t)^{\mathrm{T}} \geqslant \delta I>0$ для некоторого $\delta>0$ при всех $x, t$.

Известно [8, § II.6], что при таких предположениях уравнение (1.1)(1.2) имеет слабое решение (однозначное по распределению). Введенный здесь набор $(\Omega, \mathscr{F}, \mathbf{P}, w(t), y(t))$ является, таким образом, одним из таких слабых решений.

Пусть $D \subset \mathbf{R}^{n}$ - некоторая область. Полагаем, что либо $D=\mathbf{R}^{n}$, либо область $D$ ограничена и имеет $C^{2}$-гладкую границу, $a \in D$ п.н.

Рассмотрим случайные моменты первого выхода $\tau_{D}=\inf \{t: y(t) \notin$ $D\}$. Пусть для п.в. (почти всех) $t \in[0, T]$ задана ограниченная гиперповерхность $\Gamma(t) \subset D$ размерности $n-1, \partial \Gamma(t)$ - край этой гитерповерхности. Введем множества

$$
\begin{aligned}
& \Gamma_{*}(t, \varepsilon)=\left\{x \in D: \inf _{y \in \Gamma(t)}|x-y|<\frac{\varepsilon}{2}\right\}, \\
& \Gamma^{\prime}(t, \varepsilon)=\left\{x \in D: \inf _{y \in \partial \Gamma(t)}|x-y|<\frac{\varepsilon}{2}\right\} .
\end{aligned}
$$

(Возможно, $\partial \Gamma(t)=\varnothing$, тогда $\Gamma^{\prime}(t, \varepsilon)=\varnothing$.) Для всех $t \in[0, T], \varepsilon>0$ зададим произвольным образом борелевские множества $\Gamma(t, \varepsilon)$ такие, что

$$
\Gamma_{*}(t, \varepsilon) \backslash \Gamma^{\prime}(t, \varepsilon) \subseteq \Gamma(t, \varepsilon) \subseteq \Gamma_{*}(t, \varepsilon)
$$

Нас будет интересовать локальное время пребывания процесса $y(t)$ на $\Gamma(t)$ (до выхода из $D$ ), т.е. предельные значения при $\varepsilon \rightarrow+0$ величин

$$
l_{\varepsilon}(t)=\frac{1}{\varepsilon} \int_{0}^{\tau_{D} \wedge t} \operatorname{Ind}\{y(s) \in \Gamma(s, \varepsilon)\} d s .
$$


В случае $f, \beta, \Gamma(t)$ общего вида будет показано, что для любого $t>0$ эти величины сходятся в среднеквадратическом к некоторой случайной величине $\hat{\mathbf{t}}(t)$. Сходимость по распределению установлена в п. 5 , в среднеквадратическом - в п. 6 (при этом предел не зависит от выбора $\Gamma(t, \varepsilon)$ в (1.4)). Будет найдено уравнение для характеристической функции

$$
\phi(z)=\mathbf{E} e^{z \hat{z}(t)}, \quad z \in \mathbf{C} .
$$

Кроме того, будут выведены уравнения, являюшиеся аналогом обратного уравнения Колмогорова (и установлена их разрешимость в соответствуюших классах функций) для функционалов типа

$$
\phi_{1}=\mathbf{E} \hat{\mathrm{t}}(T), \quad \phi_{2}=\mathbf{E} \int_{0}^{\tau_{D} \wedge T} \varphi(y(t), t) e^{\hat{\mathrm{t}}(t)} d t
$$

для заданного числа $T>0$ и функции $\varphi$.

2. Пространства и классы функций. Зададим число $T>0$. Обозначим $Q=D \times(0, T)$. Ниже $\|\cdot\|_{X}$ означает норму в пространстве $X,(\cdot, \cdot)_{X}$ есть скалярное произведение в гильбертовом пространстве $X$.

Введем пространства (комплекснозначных) функций. Для целых $k \geqslant 0$ пусть $H^{k}=\stackrel{\circ}{W}_{2}^{k}(D)$ - гильбертовы пространства Соболева, $H^{-k}$ - пространство, двойственное к $H^{k}$, с нормой $\|\cdot\|_{H^{-1}}$ такой, что $\|u\|_{H^{-1}}$ для $u \in H^{0}$ есть супремум $(u, v)_{H^{0}}$ пाо $v \in H^{0},\|v\|_{H^{k}} \leqslant 1$.

Пусть $\ell_{m}$ означает меру Лебега в $\mathbf{R}^{m}, \overline{\mathscr{B}}_{m}$ есть $\sigma$-алгебра лебеговских множеств в $\mathbf{R}^{m}$. Введем пространства $\mathscr{C}^{k}=C\left([0, T] \rightarrow H^{k}\right), X^{k}=$ $L^{2}\left([0, T], \overline{\mathscr{B}}_{1}, \ell_{1}, H^{k}\right), Y^{k}=X^{k} \cap \mathscr{C}^{k-1}$ с нормой $\|u\|_{Y^{k}}=\|u\|_{X^{k}}+\|u\|_{\mathscr{C}^{k-1}}$.

Форму $(u, v)_{H^{0}}$ считаем определенной также и для $u \in H^{-k}, v \in H^{k}$ (распространяя ее естественным образом с $u \in H^{0}, v \in H^{k}$ ).

Пусть ниже $\mu \in(1,2)$ - произвольное число в случае $n=1$,

$$
\mu \in\left(1, \frac{n}{n-1}\right) \quad \text { в случае } n>1 .
$$

Введем пространство $\mathscr{W}=W_{\mu}^{(1)}(D)$ и сопряженное пространство $\mathscr{W}^{*}$, а также пространство $\mathscr{X}=L^{\infty}\left([0, T], \overline{\mathscr{B}}_{1}, \ell_{1}, \mathscr{W}^{*}\right)$.

Следующее предложение стандартно.

Предложение 2.1. i) Если $D$ - ограниченная область, то $\mathscr{W}^{*} \subset$ $H^{-1},\|g\|_{H^{-1}} \leqslant c\|g\|_{W^{*}}$, где $c=c(n, D, \mu)-$ константа, $и \mathscr{X} \subset X^{-1}$.

ii) Пусть $D=\mathbf{R}^{n}, g \in \mathscr{W}^{*}$ и существует такая ограниченная область $D_{1} \subset \mathbf{R}^{n}$, чтоо $\langle\xi, g\rangle=0\left(\forall \xi \in \mathscr{W}: \operatorname{supp} \xi \subset \mathbf{R}^{n} \backslash D_{1}\right)$. Toгda $g \in H^{-1},\|g\|_{H^{-1}} \leqslant c\|g\|_{W^{*}}$, где $c=c(n, D, \mu)-$ константа.

Лемма 2.1. Для $\xi \in H^{1}, \eta \in H^{1}$ выполнено $\xi \eta \in \mathscr{W} u\|\xi \eta\|_{W} \leqslant$ $c\|\xi\|_{H^{1}}\|\eta\|_{H^{1}}$, где $c=c(n, D, \mu)-$ константа. 
Д ока з а т ель с т в о. Пусть $r=2 / \mu, r^{\prime}=r(r-1)^{-1}, p=\mu r^{\prime}$, $\bar{\eta} \in H^{0}$. Имеем $p=\mu r^{\prime}=2 \mu(2-\mu), \mu(2-\mu)^{-1}<n(n-2)^{-1}, p<2 n(n-2)^{-1}$ при $n>2$,

$$
\begin{aligned}
\|\xi \bar{\eta}\|_{L_{\mu}(D)} & =\left(\int_{D} \xi^{\mu} \bar{\eta}^{\mu} d x\right)^{1 / \mu} \leqslant\left(\left(\int_{D} \xi^{\mu r^{\prime}} d x\right)^{1 / r^{\prime}}\left(\int_{D} \bar{\eta}^{2} d x\right)^{1 / r}\right)^{1 / \mu} \\
& =\|\bar{\eta}\|_{H^{\circ}}\left(\int_{D} \xi^{p} d x\right)^{1 / p} \leqslant c\|\bar{\eta}\|_{H^{\circ}}\|\xi\|_{H^{1}}
\end{aligned}
$$

где константа $c=c(n, D, \mu)$. Последняя оценка получена из теорем вложения для пространств Соболева (см. [5, с. 78]). Применив (2.2) к $\left(\partial \xi / \partial x_{i}\right) \eta,\left(\partial \eta / \partial x_{i}\right) \xi$, получаем требуемое.

Лемма 2.2. Для $\xi \in H^{1}, g \in \mathscr{W}^{*} \cap H^{0}$ выполнено

$$
\xi g \in H^{-1} \quad \text { u } \quad\|\xi g\|_{H^{-1}} \leqslant c\|\xi\|_{H^{1}}\|g\|_{W^{*}}
$$

где $c=c(n, D, \mu)-$ константа.

Доказате льство. Введем множество $B H^{1}=\left\{\eta \in H^{1}\right.$ п $\left.L_{\infty}(D):\|\eta\|_{H^{1}} \leqslant 1\right\}$. Имеем

$$
\begin{aligned}
\|\xi g\|_{H^{-1}} & =\sup _{\eta \in B H^{1}}(\eta, \xi g)_{H^{0}} \leqslant \sup _{\eta \in B H^{1}}(\eta \xi, g)_{H^{0}} \leqslant \sup _{\eta \in B H^{1}}\|\eta \xi\|_{W^{\prime}}\|g\|_{\mathscr{W}^{*}} \\
& \leqslant c \sup _{\eta \in B H^{1}}\|\eta\|_{H^{1}}\|\xi\|_{H^{1}}\|g\|_{W^{*}} \leqslant c\|\xi\|_{H^{1}}\|g\|_{W^{*}}
\end{aligned}
$$

где $c$ - константа из леммы 2.1. Отсюда получаем требуемое.

Введем параметр

$$
\mathscr{P}=\left\{n, T, D, \delta, \sup _{x, t}|f(x, t)|, \sup _{x, t}|\beta(x, t)|, \sup _{x, t, i}\left|\frac{\partial \beta(x, t)}{\partial x_{i}}\right|\right\}
$$

3. Параболическое уравнение с коэффициентом из $\mathscr{X}$. Введем и исследуем параболические уравнения, которые далее используются для описания эволющии функционалов (1.6)-(1.7) и являются аналогами обратного уравнения Колмогорова.

Введем оператор $\mathscr{A}$ :

$$
\mathscr{A} V=\sum_{i, j=1}^{n} b_{i j}(x, t) \frac{\partial^{2} V}{\partial x_{i} \partial x_{j}}(x)+\sum_{i=1}^{n} f_{i}(x, t) \frac{\partial V}{\partial x_{i}}(x) .
$$

Здесь $b_{i j}, f_{i}, x_{i}$ - компоненты матрицы $b$ и векторов $f, x$ соответственно. Будем рассматривать краевые задачи

$$
\frac{\partial V}{\partial t}+\mathscr{A} V+g V=-\varphi,\left.\quad V(x, t)\right|_{x \in \partial D}=0, V(x, T)=R(x) .
$$


Известно [5], что в случае $g \in L_{\infty}(Q), \varphi \in X^{-1}, R \in H^{0}=L_{2}(D)$ эта задача однозначно разрешима в классе $Y^{1}$.

Теорема 3.1. Пусть заданы $z \in \mathbf{C}, h \in \mathscr{X}, \varphi \in X^{-1}, R \in H^{0}$. IIyсmь $g_{\varepsilon} \in L_{\infty}(Q), g \in \mathscr{X}, h_{\varepsilon} \in L_{\infty}(Q), \varphi_{\varepsilon} \in L_{\infty}(Q), R_{\varepsilon} \in H^{0}-$ maкue функиии, что $g_{\varepsilon}=z h_{\varepsilon}, g=z h,\left\|h_{\varepsilon}-h\right\|_{\mathscr{X}} \rightarrow 0,\left\|\varphi_{\varepsilon}-\varphi\right\|_{X^{-1}} \rightarrow 0, \| R_{\varepsilon}-$ $R \|_{H^{0}} \rightarrow 0, \varepsilon \rightarrow 0$. Пусть $V_{\varepsilon}-$ решения задачи (3.2), соответствуюшие $g=g_{\varepsilon}, \varphi=\varphi_{\varepsilon}$. Тогда последовательность $V_{\varepsilon}$ имеет при $\varepsilon \rightarrow+0$ предел $V$ в $Y^{1}$, равномерный по $z:|z| \leqslant 1$, определяемый однозначно, и при этом

$$
\|V\|_{Y^{1}} \leqslant c\left(\|\varphi\|_{X^{-1}}+\|R\|_{H^{0}}\right)
$$

где $c>0$ - константа, зависящая от параметров $\mathscr{P}, \mu,\|g\|_{\mathscr{X}}$.

Отметим, что $V$ в теореме для любого заданного $g$ линейно зависит от $\varphi, R$. Кроме того, $V=0$ в случае $\varphi=0, R=0$. Отсюда следует, что линеен и однороден оператор, сопоставляющий решение $V$ паре $(\varphi, R) \in X^{-1} \times H^{0}$. Введем операторы $L(g), \mathscr{L}(g)$ такие, что $V=L(g) \varphi+\mathscr{L}(g) R$ для соответствуюшего значения $V$ из теоремы 3.1 . По теореме, $L(g): X^{-1} \rightarrow Y^{1}, \mathscr{L}(g): H^{0} \rightarrow Y^{1}-$ линейные непрерывные операторы; по лемме $2.2, g V \in X^{-1}$.

О п р е д е л е н и е 3.1 . Говорим, что $V$ есть решение в классе $Y^{1}$ задачи (3.2) с обобщенным $g \in \mathscr{X}$.

Д о к а з а т е л с т в о т е о е м ы 3.1. Сначала покажем, что при подстановке $g=g_{\varepsilon}$ в (3.2) константа в неравенстве (3.3) для $V=V_{\varepsilon}$ не увеличивается при $\varepsilon \rightarrow+0$.

Ниже используем элементарное неравенство

$$
u v \leqslant \frac{u^{2}}{2 \gamma}+\frac{v^{2} \gamma}{2} \quad(\forall u, v, \gamma \in \mathbf{R}, \gamma>0) .
$$

Пусть $v \in H^{1} \cap C^{2}(D)$. Имеем для любого $t \in[0, T]$ и оператора $\mathscr{A}=$ $\mathscr{A}(x, t)$ определенного в $(3.1)$, оценки

$$
\begin{aligned}
(v, \mathscr{A} v)_{H^{0}}= & \left(v, \sum_{i, j=1}^{n} b_{i j} \frac{\partial^{2} v}{\partial x_{i} \partial x_{j}}\right)_{H^{0}}+\left(v, \sum_{i=1}^{n} f_{i} \frac{\partial v}{\partial x_{i}}\right)_{H^{0}} \\
= & -\sum_{i, j=1}^{n}\left(\frac{\partial v}{\partial x_{i}}, b_{i j} \frac{\partial v}{\partial x_{j}}\right)_{H^{0}}-\sum_{i, j=1}^{n}\left(v, \frac{\partial b_{i j}}{\partial x_{i}} \frac{\partial v}{\partial x_{j}}\right)_{H^{0}} \\
& +\sum_{i=1}^{n}\left(v, f_{i} \frac{\partial v}{\partial x_{i}}\right)_{H^{0}} \\
\leqslant & -\delta \sum_{i=1}^{n}\left\|\frac{\partial v}{\partial x_{i}}\right\|_{H^{0}}^{2}+\sum_{i, j=1}^{n}\|v\|_{H^{\circ}}\left\|\frac{\partial b_{i j}}{\partial x_{i}}\right\|_{L_{\infty}(Q)}\left\|\frac{\partial v}{\partial x_{j}}\right\|_{H^{0}} \\
& +\sum_{i=1}^{n}\|v\|_{H^{0}}\left\|f_{i}\right\|_{L_{\infty}(Q)}\left\|\frac{\partial v}{\partial x_{i}}\right\|_{H^{\circ}}
\end{aligned}
$$




$$
\begin{aligned}
\leqslant & -\delta \sum_{i=1}^{n}\left\|\frac{\partial v}{\partial x_{i}}\right\|_{H^{0}}^{2}+\frac{\delta}{4} \sum_{i=1}^{n}\left\|\frac{\partial v}{\partial x_{i}}\right\|_{H^{0}}^{2} \\
& +\frac{C}{\delta} \sum_{i, j=1}^{n}\|v\|_{H^{0}}^{2}\left(\left\|\frac{\partial b_{i j}}{\partial x_{i}}\right\|_{L_{\infty}(Q)}^{2}+\left\|f_{i}\right\|_{L_{\infty}(Q)}^{2}\right)
\end{aligned}
$$

где константа $C=C(n)$. Отсюда получаем, что для любого $v \in H^{1}$ при любом $t \in[0, T]$ выполнено

$$
(v, \mathscr{A} v)_{H^{0}} \leqslant-\frac{3 \delta}{4} \sum_{i=1}^{n}\left\|\frac{\partial v}{\partial x_{i}}\right\|_{H^{0}}^{2}+C_{1}\|v\|_{H^{0}}^{2},
$$

где константа $C_{1}$ зависит от параметра $\mathscr{P}$.

Для любого $v \in H^{1}$ при любом $t \in[0, T]$ выполнено

$$
\begin{aligned}
\left(v, \varphi_{\varepsilon}(\cdot, t)\right)_{H^{0}} & \leqslant\|v\|_{H^{1}}^{2}\left\|\varphi_{\varepsilon}(\cdot, t)\right\|_{H^{-1}}^{2} \leqslant C_{D} \sum_{i=1}^{n}\left\|\frac{\partial v}{\partial x_{i}}\right\|_{H^{0}}\left\|\varphi_{\varepsilon}(\cdot, t)\right\|_{H^{-1}} \\
& \leqslant \frac{\delta}{4} \sum_{i=1}^{n}\left\|\frac{\partial v}{\partial x_{i}}\right\|_{H^{0}}^{2}+C_{2}\left\|\varphi_{\varepsilon}(\cdot, t)\right\|_{H^{-1}}^{2}
\end{aligned}
$$

где константа $C_{D}$ зависит от $n, D$, константа $C_{2}$ зависит от $\mathscr{P}$.

Для любого $v \in H^{1}$ выполнено

$$
\begin{aligned}
\left(v, g_{\varepsilon} v\right)_{H^{0}} & \leqslant\left\|v^{2}\right\|{ }_{W}\left\|g_{\varepsilon}\right\|_{W^{*}} \leqslant C_{3}\|v\|_{H^{1}}\|v\|_{H^{0}}\left\|g_{\varepsilon}\right\| W_{W^{*}} \\
& \leqslant \frac{\delta}{4} \sum_{i=1}^{n}\|v\|_{H^{1}}^{2}+\widehat{C}_{3}\|v\|_{H_{0}}^{2} \leqslant \frac{\delta}{4} \sum_{i=1}^{n}\left\|\frac{\partial v}{\partial x_{i}}\right\|_{H^{0}}^{2}+C_{3}\|v\|_{H_{0}}^{2}
\end{aligned}
$$

где константы $\widehat{C}_{3}, C_{3}$ зависят от $\sup _{\varepsilon}\left\|g_{\varepsilon}\right\|_{\mathscr{X}}, \delta, n, D$.

Для решения $V=V_{\varepsilon}$ задачи (3.2) с $g=g_{\varepsilon}, \varphi=\varphi_{\varepsilon}, \varepsilon \in\left(0, \varepsilon_{1}\right]$ имеем в силу $(3.5)-(3.7)$

$$
\begin{aligned}
& \left\|V_{\varepsilon}(\cdot, t)\right\|_{H^{0}}^{2}-\left\|V_{\varepsilon}(\cdot, T)\right\|_{H^{0}}^{2} \\
& =2 \int_{t}^{T}\left(V_{\varepsilon}(\cdot, s), \mathscr{A} V_{\varepsilon}(\cdot, s)+g_{\varepsilon} V_{\varepsilon}(\cdot, s)+\varphi_{\varepsilon}(\cdot, s)\right)_{H^{\circ}} d s \\
& \leqslant \int_{t}^{T}\left\{-\delta \sum_{i=1}^{n}\left\|\frac{\partial V_{\varepsilon}}{\partial x_{i}}(\cdot, s)\right\|_{H^{0}}^{2}\right. \\
& \left.+C_{4}\left(\left\|V_{\varepsilon}(\cdot, s)\right\|_{H^{0}}^{2}+\left\|\varphi_{\varepsilon}(\cdot, s)\right\|_{H^{-1}}^{2}\right)\right\} d s
\end{aligned}
$$

где константа $C_{4}$ зависит от $\mathscr{P}, \mu,\|g\|_{\mathscr{X}}$.

Отсюда сразу видно, что

$$
\left\|V_{\varepsilon}\right\|_{Y^{1}} \leqslant C_{*}\left(\left\|\varphi_{\varepsilon}\right\|_{X^{-1}}+\|R\|_{H^{0}}\right) \quad\left(\forall \varepsilon \in\left(0, \varepsilon_{1}\right]\right)
$$

где константа $C_{*}$ зависит от $\mathscr{P}, \mu,\|g\|_{\mathscr{X}}$. 
Покажем, что последовательность $\left\{V_{\varepsilon}\right\}$ сходится в себе в $Y^{1}$. Пусть $\varepsilon_{1} \rightarrow 0, \varepsilon_{2} \rightarrow 0$. Обозначим $W=V_{\varepsilon_{1}}-V_{\varepsilon_{2}}$. Имеем

$$
\frac{\partial W}{\partial t}+\mathscr{A} W+g_{e_{1}} W=-\xi,\left.\quad W(x, t)\right|_{x \in \partial D}=0, W(x, T)=0,
$$

где $\xi=\varphi_{\varepsilon_{1}}-\varphi_{\varepsilon_{2}}+\left(g_{\varepsilon_{1}}-g_{\varepsilon_{2}}\right) V_{\varepsilon_{2}}$. Имеем

$$
\left\|\varphi_{\varepsilon_{1}}-\varphi_{\varepsilon_{2}}\right\|_{X^{-1}} \longrightarrow 0, \quad\left\|g_{\varepsilon_{1}}-g_{\varepsilon_{2}}\right\|_{\mathscr{C}} \longrightarrow 0,
$$

поскольку последовательности $\left\{\varphi_{\varepsilon_{i}}\right\},\left\{g_{\varepsilon_{i}}\right\}$ сходятся в себе и сходятся в соответствующих пространствах. По лемме 2.2 ,

$$
\begin{aligned}
\left\|\left(g_{\varepsilon_{1}}-g_{\varepsilon_{2}}\right) V_{\varepsilon_{2}}\right\|_{X^{-1}} & =\int_{0}^{T}\left\|\left(g_{\varepsilon_{1}}-g_{\varepsilon_{2}}\right) V_{\varepsilon_{2}}(\cdot, t)\right\|_{H^{-1}} d t \\
& \leqslant \int_{0}^{T}\left\|g_{\varepsilon_{1}}-g_{\varepsilon_{2}}\right\| W_{W^{*}}\left\|V_{\varepsilon_{2}}(\cdot, t)\right\|_{H^{1}} d t \\
& \leqslant\left\|g_{\varepsilon_{1}}-g_{\varepsilon_{2}}\right\|_{\mathscr{X}} \int_{0}^{T}\left\|V_{\varepsilon_{2}}(\cdot, t)\right\|_{H^{-1}} d t \\
& =\left\|g_{\varepsilon_{1}}-g_{\varepsilon_{2}}\right\| \mathscr{X}\left\|V_{\varepsilon_{2}}\right\|_{X^{1}} \longrightarrow 0
\end{aligned}
$$

при $\varepsilon_{1} \rightarrow 0, \varepsilon_{2} \rightarrow 0$, ввиду (3.9), (3.11). Значит, $\|\xi\|_{X^{-1}} \rightarrow 0$. Применив оценку (3.9) к решению $W$ краевой задачи (3.10), получаем, что

$$
\|W\|_{Y^{1}} \leqslant C_{*}\|\xi\|_{X^{-1}} \longrightarrow 0 .
$$

Отсюда видно, что последовательность $\left\{V_{\varepsilon}\right\}, \varepsilon=\varepsilon_{i} \rightarrow 0$, сходится в себе (а значит, сходится) в банаховом пространстве $Y^{1}$. Оценка (3.3) следует из (3.9), из этой же оценки следует однозначность $V$.

Покажем, что последовательность $\left\{V_{\varepsilon}\right\}$ сходится в $Y^{1}$ равномерно по $z \in \mathbf{C}:|z| \leqslant 1$. Пусть $\varepsilon \rightarrow 0$. Обозначим $W=V_{\varepsilon}-V$. Имеем

$$
\frac{\partial W}{\partial t}+\mathscr{A} W+g_{\varepsilon} W=-\xi,\left.\quad W(x, t)\right|_{x \in \partial D}=0, W(x, T)=R_{\varepsilon}-R,
$$

где $\xi=\varphi_{\varepsilon}-\varphi+\left(g_{\varepsilon}-g\right) V$. Имеем

$$
\left\|\varphi_{\varepsilon}-\varphi\right\|_{X^{-1}} \longrightarrow 0, \quad\left\|g_{\varepsilon}-g\right\|_{\mathscr{X}}=z\left\|h_{\varepsilon}-h\right\|_{\mathscr{X}} \longrightarrow 0
$$

равномерно по $|z| \leqslant 1$. По лемме 2.2 ,

$$
\begin{aligned}
\left\|\left(g_{\varepsilon}-g\right) V\right\|_{X^{-1}} & =\int_{0}^{T}\left\|\left(g_{\varepsilon}-g\right) V_{\varepsilon_{2}}(\cdot, t)\right\|_{H^{-1}} d t \\
& \leqslant \int_{0}^{T}\left\|g_{\varepsilon}-g\right\|\left\|_{W^{*}}\right\| V(\cdot, t) \|_{H^{1}} d t \\
& \leqslant\left\|g_{\varepsilon}-g\right\|_{\mathscr{X}} \int_{0}^{T}\|V(\cdot, t)\|_{H^{1}} d t \\
& =\left\|g_{\varepsilon}-g\right\|_{\mathscr{X}}\|V\|_{X^{1}} \longrightarrow 0
\end{aligned}
$$


при $\varepsilon \rightarrow 0$ и $\|\xi\|_{X^{-1}} \rightarrow 0$ равномерно по $|z| \leqslant 1$. Применив оценку (3.3) к решению $W$ краевой задачи (3.14), получаем, что

$$
\|W\|_{Y^{1}} \leqslant C_{*}\left(\|\xi\|_{X^{-1}}+\left\|R_{\varepsilon}-R\right\|_{H^{0}}\right) \longrightarrow 0 .
$$

Отсюда видно, что последовательность $\left\{V_{\varepsilon}\right\}, \varepsilon \rightarrow 0$, сходится равномерно по $|z| \leqslant 1$. Теорема 3.1 доказана.

4. О классе гиперповерхностей. Естественно предположить, что для изучения функционалов типа (1.6)-(1.7) пригодны уравнения (3.2) с такими $g \in \mathscr{X}, \varphi \in X^{-1}$, что в качестве функций $g_{\varepsilon}, \varphi_{\varepsilon}$, фигуриругших в теореме 3.1 , можно взять функции $\varepsilon^{-1} \operatorname{Ind}\{x \in \Gamma(t, \varepsilon)\}$ для гиперповерхности $\Gamma(t) \subset D$ (множества $\Gamma(t, \varepsilon)$ определены в (1.3)-(1.4)). Ниже будет показано, что предел $\varepsilon^{-1} \operatorname{Ind}\{x \in \Gamma(t, \varepsilon)\}$ принадлежит и $X^{-1}$ и $\mathscr{X}$ для $\Gamma(t)$ из достаточно широкого класса «кусочно $C^{1}$-гладких» гиперповерхностей.

Через $e^{(j)}$ обозначаем $j$-й орт в $\mathbf{R}^{n}$.

Пусть $\Gamma$ - некоторая поверхность. Обозначим через $N(x, j)$ число пересечений гиперповерхности $\Gamma$ лучом из $x=\left(x_{1}, x_{2}, \ldots, x_{n}\right)$ в $\left(x_{1}, \ldots, x_{j-1},-\infty, x_{j+1}, \ldots, x_{n}\right)$, точки $\widehat{x}_{k}(x, j)$ являются соответствующими точками пересечения; полагаем $N(x, j)=+\infty$, если луч проходит по касательной к $Г$.

Введем функции $\gamma_{j}: \Gamma \rightarrow \mathbf{R}$ такие, что $\gamma_{j}(x)=\left|\cos \alpha_{j}(x)\right|$, где $\alpha_{j}(x)$ - угол между $e^{(j)}$ и нормалью к Г в точке $x \in \Gamma$, если эта нормаль определена; $\gamma_{j}(x)=0(\forall j)$, если $x-$ точка нарушения гладкости, где не определена нормаль. Определим функции

$$
G_{j}(x)=\sum_{k=1}^{N(x, j)} \gamma_{j}\left(\widehat{x}_{k}(x, j)\right) .
$$

Полагаем $G_{j}(x)=+\infty$, если $N(x, j)=+\infty$.

Теорема 4.1. Пусть задано миожество $\widehat{\Gamma} \subset \mathbf{R}^{n}$, являющееся объединением конечного числа $\mathcal{N}$ многогранников $\widehat{\Gamma}_{i}$ размерности $n-1$ с попарно непересекаючимися внутренностями, $\widehat{\Gamma}=\cup_{i=1}^{\mathcal{N}} \widehat{\Gamma}_{i}$. Пусть $\mathscr{B}: \mathbf{R}^{n} \rightarrow \mathbf{R}^{n}-$ некоторая непрерывная биективная бункция, гиперповерхность $\Gamma \subset D$ такова, ито $\Gamma=\mathscr{M}(\widehat{\Gamma})$. Iредполагаем, ито функчии $\mathscr{B}: \widehat{\Gamma}_{i} \rightarrow \mathbf{R}^{n}$ являются $C^{1}-$ гладкими биекчиями, $\mathscr{B}(x)=x$, если $x$ является вершиной какого-либо $\widehat{\Gamma}_{i},\left|\mathrm{n}(x)-\mathrm{n}_{i}\right| \leqslant \delta_{0}$, если $x$ принадлежит внутренней части $\Gamma_{i}, i=1, \ldots, \mathcal{N}$. Здесь $\Gamma_{i}=\mathscr{B}\left(\widehat{\Gamma}_{i}\right), \mathbf{n}(x)-$ нормаль $\kappa$ Г в $x, \mathbf{n}_{i}$ - нормаль $x \widehat{\Gamma}_{i}, \delta_{0} \leqslant n^{-2} / 2$ (ориектачия кормалей фиксирована, $\left.|\mathbf{n}(x)|=1,\left|\mathbf{n}_{i}\right|=1\right)$. Тогда $N(x, j)<+\infty$ для всех $j$ для п.в. $x$. Oпределим обобщенные функции

$$
g=\sum_{j=1}^{n} \frac{\partial G_{j}}{\partial x_{j}}
$$


Tогда $g \in \mathscr{W}^{*} \cap H^{-1}$, функции $g_{\varepsilon}(x)=\varepsilon^{-1} \operatorname{Ind}\{x \in \Gamma(\varepsilon)\}$ сходятся $\kappa g$ в мeтpuкe $\mathscr{W}^{*} u H^{-1}, u$

$$
\|g\|_{W^{*}} \leqslant c \sum_{j=1}^{n}\left\|G_{j}\right\|_{L_{\nu}\left(D_{1}\right)}, \quad\|g\|_{H^{-1}} \leqslant c \sum_{j=1}^{n}\left\|G_{j}\right\|_{L_{2}\left(D_{1}\right)},
$$

где $\nu=\mu(\mu-1)^{-1}, D_{1}$ - ограниченная область в $\mathbf{R}^{n}$, такая, что $\Gamma \subset D_{1} \subseteq D, c=c\left(n, D_{1}\right)-$ константа.

3 а м е ч а н и е 4.1. Нетрудно заметить, что предположения теоремы 4.1 выполнены для дисков, сфер и многих других кусочно $C^{1}$. гладких $(n-1)$-мерньіх поверхностей.

Док аз а те лг с т во те о ре м ы 4.1. Обозначим $S_{0}=\cup \partial \Gamma_{i}$; имеем $\ell_{n}\left(S_{0}\right)=0$. Рассмотрим множество открытых областей $D_{i} \subseteq D$ таких, что $\bar{D}=\cup_{i=1}^{\mathscr{N}} \bar{D}_{i}, D_{i} \cap D_{j}=\varnothing$, если $i \neq j, \Gamma_{i}=\Gamma \cap \bar{D}_{i}$ (через $\bar{D}_{i}, \bar{D}$ обозначаем замыкания областей). Обозначим $\Gamma_{i}(\varepsilon)=\Gamma(\varepsilon) \cap \bar{D}_{i}$. Обозначим $P_{j}(x)$ прямую линию, проходящую через $x$ и параллельную $e^{(j)}$. Зададим функции $\psi(\cdot, \varepsilon): \Gamma(\varepsilon) \longrightarrow \Gamma \backslash S_{0}$ такие, что $\psi(x) \in \Gamma_{i}$, если $x \in \Gamma_{i}(\varepsilon),|\psi(x, \varepsilon)-x| \leqslant c \varepsilon$, где константы $c$ таковы, что эти функции существуют для всех $\varepsilon \leqslant \varepsilon_{*}$ для некоторого малого $\varepsilon_{*}>0$. Зададим функции $G_{j}^{\varepsilon}(x), \boldsymbol{x}=\left(x_{1}, \ldots, x_{n}\right)$. Полагаем, что эти функции непрерывны по $x_{j}$. Полагаем, что эти функции $G_{j}^{\varepsilon}(x)$ постоянны на каждом отрезке $P_{j}(x)$, лежащем в $D \backslash \Gamma(\varepsilon)$ (константы свои для каждого такого отрезка), $G_{j}^{\varepsilon}(x)=0$, если $\left(x_{1}, \ldots, x_{j-1}, y_{j}, x_{j+1}, \ldots, x_{n}\right) \notin \Gamma(\varepsilon)\left(\forall y_{j}<x_{j}\right)$, и $\partial G_{j}^{\varepsilon}(x) / \partial x_{j}=\varepsilon^{-1} \gamma_{j}^{2}(\psi(x, \varepsilon)), x \in P_{j}(x) \cap \Gamma(\varepsilon)$. Эти условия определяют $G_{j}^{\varepsilon}(x)$ однозначно.

Имеем $\sum_{j=1}^{n} \gamma_{j}^{2}(x)=1\left(\forall x \in \Gamma \backslash S_{0}\right)$. Тогда

$$
\begin{aligned}
& \sum_{j=1}^{n} \frac{\partial G_{j}^{\varepsilon}}{\partial x_{j}}(x)=\frac{1}{\varepsilon} \sum_{j=1}^{n} \gamma_{j}^{2}(\psi(x, \varepsilon))=\frac{1}{\varepsilon}, \quad x \in \Gamma(\varepsilon), \\
& \sum_{j=1}^{n} \frac{\partial G_{j}^{\varepsilon}}{\partial x_{j}}(x)=0, \quad x \notin \Gamma(\varepsilon), \quad \sum_{j=1}^{n} \frac{\partial G_{j}^{\varepsilon}}{\partial x_{j}}(x)=g_{\varepsilon}(x), \quad x \in D .
\end{aligned}
$$

Имеем $\ell_{n}\left(x \in \mathbf{R}^{n}: P_{j}(x) \cap S_{0} \neq \varnothing\right)=0, j=1, \ldots, n$.

По предложению 2.1 [7], выполнено $N(j, x)<+\infty$ для п.в. $x$ (можно представить луч как решение тривиального обыкновенного дифференциального уравнения, тогда предложение 2.1 [7] можно переформулировать для данного случая). Ввиду кусочной $C^{1}$-гладкости $\Gamma$ имеем $N(x, j) \leqslant$ const для п.в. $x$.

Пусть $\mathcal{N}=1, \Gamma=\Gamma_{1}$ таково, что $\mathbf{n}_{1}=\left(\mathbf{n}_{1}^{(1)}, \ldots, \mathbf{n}_{1}^{(n)}\right),\left|\mathbf{n}_{1}^{(j)}\right|=n^{-1 / 2}$, $j=1, \ldots, n$, тогда $\gamma_{j}(x) \geqslant \delta_{1}\left(\forall x \in \Gamma \backslash S_{0}\right)$ для некоторых $\delta_{1}>0$. Обозначим через $\mu_{j}(x, \varepsilon)$ длину минимального отрезка, лежащего в $\Gamma(\varepsilon)$, с концами в $\partial \Gamma(\varepsilon)$, проходящего через $x$ и параллельного $e^{(j)}$. Име- 
ем $\varepsilon^{-1} \mu_{j}(x, \varepsilon) \longrightarrow \gamma_{j}^{-1}(x)$. Из непрерывности нормали на данной $C^{1}-$ гладкой поверхности $\Gamma=\Gamma_{1}$ следует, что $G_{i}^{\varepsilon}(x) \rightarrow G_{i}(x)$ при $\varepsilon \rightarrow+0$ для II.в. $x, i=1, \ldots ; n$,

$$
\sup _{x}\left|G_{j}^{\varepsilon}(x)\right| \leqslant \varepsilon^{-1} \mu_{j}(x, \varepsilon) \sup _{x} \gamma_{j}^{2}(\psi(x, \varepsilon)) \leqslant c
$$

где $x \in P_{j}(x) \cap \Gamma(\varepsilon)$, константа $c$ зависит от $\Gamma$. Значит, $\left\|G_{i}^{\varepsilon}-G_{i}\right\|_{L_{\nu}(D)} \rightarrow$ 0 при $\varepsilon \rightarrow+0(\forall \nu>1, i=1, \ldots, n)$, или $g_{\varepsilon}=\sum_{j=1}^{n} \partial G_{j}^{\varepsilon} / \partial x_{j}(x) \longrightarrow g$ в $\mathscr{W}^{*}$. Пусть $u \in C^{2}(D) \cap \mathscr{W}^{*} \cap H^{1}$. Имеем

$$
\begin{aligned}
\left\langle g_{\varepsilon}-g, u\right\rangle= & \sum_{j=1}^{n} \int_{\partial D}\left(G_{j}^{\varepsilon}(x)-G_{j}(x)\right) u(x) \cos \left(\mathbf{n}(x), e_{j}\right) d x \\
& -\sum_{j=1}^{n} \int_{D}\left(G_{j}^{\varepsilon}(x)-G_{j}(x)\right) \frac{\partial u}{\partial x_{j}}(x) d x \\
= & -\sum_{j=1}^{n} \int_{D}\left(G_{j}^{\varepsilon}(x)-G_{j}(x)\right) \frac{\partial u}{\partial x_{j}}(x) d x
\end{aligned}
$$

поскольку $G_{j}^{\varepsilon} \equiv G_{j}$ на $\partial D$ (при $D=\mathbf{R}^{n}$ интегралы по $\partial D$ во втором выражении исчезают). Значит, для $\nu=\mu(\mu-1)^{-1}$ имеем

$$
\mid\left\langle g_{\varepsilon}-g, u\right| \leqslant \sum_{j=1}^{n}\left(G_{j}^{\varepsilon}-G_{j}\right)_{L_{\nu}(D)}\|u\|_{\mathscr{W}} .
$$

Следовательно, $g_{\varepsilon}=\sum_{j=1}^{n} \partial G_{j}^{\varepsilon} / \partial x_{j}(x) \longrightarrow g$ в $\mathscr{W}^{*}$. В указанном частном случае теорема 4.1 доказана.

Пусть $\mathcal{N}=1$, нормаль $\mathbf{n}_{1}$ произвольно направлена. Введем новые координаты $\widetilde{x}_{j}=\sum_{k=1}^{n} \theta_{j k} x_{k}$, где $\theta \in \mathbf{R}^{n \times n}-$ такая матрица, что $\theta^{\mathrm{T}}=$ $\theta^{-1}$ и $\left|\widetilde{\mathbf{n}}_{1}^{(j)}\right|=n^{-1 / 2}, \mathbf{n}_{1}^{(j)} \widetilde{\mathbf{n}}_{1}^{(j)} \geqslant 0, j=1, \ldots, n$, где $\widetilde{\mathbf{n}}_{1}=\left(\widetilde{\mathbf{n}}_{1}^{(1)}, \ldots, \dot{\widetilde{\mathbf{n}}}_{1}^{(n)}\right)-$ соответствуюпая нормаль к $\theta \widehat{\Gamma}$ в новых координатах. Обозначим через $\widetilde{N}(x, j)$ число пересечений гиперповерхности $\widetilde{\Gamma}=\theta \Gamma$ лучом из $\widetilde{x}=$ $\left(\widetilde{x}_{1}, \widetilde{x}_{2}, \ldots, \widetilde{x}_{n}\right)$ в $\left(\widetilde{x}_{1}, \ldots, \widetilde{x}_{j-1},-\infty, \widetilde{x}_{j+1}, \ldots, \widetilde{x}_{n}\right)$, точки $\widetilde{x}_{k}^{\prime}(x, j)$ являются соответствуюшими точками пересечения; полагаем $\widetilde{N}(x, j)=+\infty$, если луч проходит по касательной к Г. Тогда $\widetilde{N}(x, j)<+\infty$ для всех $j$ для п.в. $x$. Определим функции $\widetilde{G}_{j}(\widetilde{x})=\sum_{k=1}^{\widetilde{N}(\widetilde{x}, j)} \gamma_{j}\left(\widetilde{x}_{k}^{\prime}(\widetilde{x}, j)\right)$. Здесь $\widetilde{\gamma}_{j}(\tilde{x})$ - соответствующие функции, построенные по правилу, указанному перед формулировкой теоремы, но в новых координатах. Обозначим $\widetilde{g}=\sum_{j=1}^{n} \partial \widetilde{G}_{j} / \partial \widetilde{x}_{j}$. Как и выше, получаем, что $\widetilde{g}_{\varepsilon}(\tilde{x})=\operatorname{Ind}\{x=$ $\left.\theta^{-1} \widetilde{x} \in \Gamma(\varepsilon)\right\} \longrightarrow \widetilde{g}$ в $\mathscr{W}^{*}$. Обозначим $\Delta G_{j}(x)$ скачок функции $G_{j}$ в $x$ на прямой $P_{j}(x)$, в порядке возрастания $x_{j}$. Имеем $\Delta G_{j}(x)=\gamma_{j}(x)$. Обозначим $\Delta \widetilde{G}_{j}(\widetilde{x})$ аналогичные скачки в новых координатах. Из того, что преобразование координат является поворотом, видно, что $\Delta \widetilde{G}_{j}(\widetilde{x})=$ 
$\sum_{k=1}^{n} \theta_{j k} \Delta G_{k}\left(\theta^{-1} \widetilde{x}\right)$, если $N(x, j)<+\infty, x \in \Gamma \backslash S_{0}$. Значит, $\langle\widetilde{\xi}, \widetilde{g}\rangle=\langle\xi, g\rangle$ для $\widetilde{\xi} \in \mathscr{W}, \xi(x)=\widetilde{\xi}(\theta x)$. Следовательно, теорема 4.1 доказана в этом случае.

Пусть $\mathscr{N}>1, g_{\varepsilon}^{(i)}(x)=\operatorname{Ind}\left\{x \in \Gamma_{i}(\varepsilon)\right\}$. Имеем $g_{\varepsilon}=\sum_{i=1}^{\mathcal{N}} g_{\varepsilon}^{(i)}$,

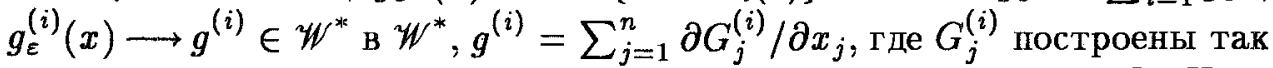
же, как в формулировке теоремы 4.1 для соответствующих $\Gamma=\Gamma_{i}$. Имеем $G_{j}(x)=\sum_{i=1}^{\mathcal{N}} G^{(i)}(x)$. Отсюда следует доказательство теоремы 4.1 .

Приведем пример поверхности $\Gamma=\Gamma(t)$, которая изменяется во времени, приближаясь к фракталу, и при этом выполнено $g=g(t) \in X^{-1}$ для $g(t)$, определенных для каждого $t$ в теореме 4.1 .

П р и м е р 4.1. Пусть $n=2, T=2$,

$$
\Gamma(t)=\left\{\left(x_{1}, x_{2}\right): x_{2}=\sin \left(x_{1}(1-t)^{-1 / 3}\right), x_{1} \in[-1,1]\right\},
$$

тогда $g=g(t) \in X^{-1}$.

5. Вероятностная интерпретация решения параболического уравнения для обобщенных $g, \varphi$. Всюду ниже полагаем, что для процесса $y(t)$ и уравнений (1.1)-(1.2) выполнены предположения $\S 1$, кроме того, предполагаем, что случайный начальный вектор $a=y(0)$ имеет плотность распределения $\rho \in H^{0}=L_{2}(D)$. Полагаем, что для п.в. $t \in[0, T]$ задана гиперповерхность $\Gamma(t)$, для которой выполнены предположения теоремы $4.1, g(t)$ - соответствуюшие элементы $\mathscr{W}^{*} \cap H^{-1}$, определенные в теореме $4.1, g-$ соответствуюший элемент $X^{-1}$. ные (1.5).

Пусть $\Gamma(t, \varepsilon)$ определены в $(1.3)-(1.4), l_{\varepsilon}(t)-$ величины, задан-

Теорема 5.1. Пусть $\rho \in L_{2}(D), g \in \mathscr{X}$.

а) Для любого $t>0$ суиествует такая случайная величина $\hat{\mathbf{t}}(t)$, что $l_{\varepsilon}(t) \rightarrow \hat{\mathbf{t}}(t)$ по распределению при $\varepsilon \rightarrow+0$.

б) Пусть $z \in \mathbf{C}$. Введем обобщенную функиию $g_{1}=z g$. Пусть $V_{1}=L\left(g_{1}\right) g, V=z V_{1}$ (иными словами, $\left.V=z L(z g) g\right)$. Тогда $V \in Y^{1}$,

$$
1+(V(\cdot, 0), \rho)_{H^{0}}=\lim _{\varepsilon \longrightarrow+0} \mathbf{E} \exp \left\{z l_{\varepsilon}(T)\right\}=\mathbf{E} \exp \{z \hat{\mathbf{t}}(T)\}
$$

причем предел имеет место равномерно по $z \in \mathbf{C}:|z| \leqslant 1$.

Следствие 5.1. В предположениях теоремы $5.1 \mathrm{E} e^{k \hat{\mathrm{t}}(t)}<+\infty$, $\mathbf{E} \hat{\mathbf{t}}(t)^{k}<+\infty(\forall k>0)$.

3 а м е ч а н и е 5.1. В работе [4] было в принципе установлено существование локального времени пребывания на гладких гиперповерхностях обших многомерных прощессов Ито. Точнее, там доказано сушествование непрерывных неубывағших процессов $\hat{\mathbf{t}}(t)$, возрастающих только на гиперповерхности и таких, что их можно получить как предел п.н. величин $\hat{l}_{\varepsilon}(t)=\int_{0}^{t} \widehat{g}_{\varepsilon}(y(t)) d t$, где $\widehat{g}_{\varepsilon}-$ некоторые неотрицательные функции; но был оставлен открытым вопрос, можно ли в общем случае 
взять $\widehat{g}_{\varepsilon}=g_{\varepsilon}$ (индикатор окрестности гиперповерхности с нормирующим множителем).

Везде ниже $\hat{\mathbf{t}}(t)$ - величины из теоремы 5.1 .

Теорема 5.2. Пусть выполнены предположения теоремы 5.1, $z \in$ $\mathbf{C}, \varphi \in X^{-1}$, функция $R \in L_{2}(D)$ измерима, $\varphi_{\varepsilon} \in L_{2}(Q)-$ измеримые функиии такие, как в теореме 3.1 для данного $\varphi, V=L(z g) \varphi+\mathscr{L}(z g) R$. Tozda

$$
\begin{aligned}
(V(\cdot, 0), \rho)_{H^{\circ}}=\lim _{\varepsilon \rightarrow+0} \mathbf{E}\{ & R(y(T)) \operatorname{Ind}\left\{\tau_{D}>T\right\} \exp \left\{z l_{\varepsilon}(T)\right\} \\
& \left.+\int_{0}^{\tau_{D} \wedge T} \exp \left\{z l_{\varepsilon}(t)\right\} \varphi_{\varepsilon}(y(t), t) d t\right\} .
\end{aligned}
$$

Дока зател льст в о те о ре м ы 5.2 . 'Введем функции $R_{\varepsilon} \in$ $C^{2}(D)$ такие, что $\left\|R_{\varepsilon}-R\right\|_{H^{\circ}} \rightarrow 0$. Введем функции $g_{\varepsilon}(x, t)=\varepsilon^{-1}$ Ind $\{x \in$ $\Gamma(t, \varepsilon)\}, V_{\varepsilon}=L\left(z g_{\varepsilon}\right) \varphi+\mathscr{L}\left(z g_{\varepsilon}\right) R_{\varepsilon}$. Имеем $V_{\varepsilon} \in W_{q}^{2,1}(Q), \forall q>1$, в силу $[5, \S \mathrm{IV} .9]$. Введем функцию

$$
W(t)=V_{\varepsilon}(y(t), t) \exp \left\{z \int_{0}^{t} g_{\varepsilon}(y(r), r) d r\right\}
$$

Имеем

$$
d_{t} W(t)=\exp \left\{z \int_{0}^{t} g_{\varepsilon}(y(r), r) d r\right\} d_{t} V_{\varepsilon}(y(t), t)+z g_{\varepsilon}(y(r), r) W(t) d t
$$

Поскольку $V_{\varepsilon} \in W_{q}^{2,1}(Q), \forall q>1$, для $d_{t} V_{\varepsilon}(y(t), t)$ можно применить формулу Ито §II.10 [8]. Используя эту формулу, получаем.

$$
\begin{aligned}
\mathbf{E}\left(W\left(\tau_{D} \wedge T\right)-W(0)\right)=\mathbf{E}\left(W\left(\tau_{D} \wedge T\right)-V_{\varepsilon}(y(0), 0)\right) \\
=-\mathbf{E} \int_{0}^{\tau_{D} \wedge T} \exp \left\{z \int_{0}^{t} g_{\varepsilon}(y(r), r) d r\right\} \varphi_{\varepsilon}(y(t), t) d t
\end{aligned}
$$

Значит,

$$
\begin{aligned}
\mathbf{E} V_{\varepsilon}(y(0), 0)=\left(V_{\varepsilon}(\cdot, 0), \rho\right)_{H^{0}} \\
=\mathbf{E}\left\{R_{\varepsilon}(y(T)) \operatorname{Ind}\left\{\tau_{D}>T\right\} \exp \left(z \int_{0}^{\tau_{D} \wedge T} g_{\varepsilon}(y(t), t) d t\right)\right. \\
\left.+\int_{0}^{\tau_{D} \wedge T} \exp \left\{z \int_{0}^{t} g_{\varepsilon}(y(r), r) d r\right\} \varphi_{\varepsilon}(y(t), t) d t\right\}
\end{aligned}
$$

По теореме 3.1 имеем $V_{\varepsilon} \rightarrow V$ в $Y^{1}$. Отсюда получаем требуемое. 
Д о к а 3 а т е ль с т в о т е о ре мы 5.1. Докажем сначала п. б). Пусть $g_{\varepsilon}(x, t)=\varepsilon^{-1}$ Ind $\{x \in \Gamma(t, \varepsilon)\}, V_{\varepsilon}=z L\left(z g_{\varepsilon}\right) g_{\varepsilon}$. Это означает, что $V_{\varepsilon}$ является решением задачи

$$
\frac{\partial V_{\varepsilon}}{\partial t}+\mathscr{A} V_{\varepsilon}+z g_{\varepsilon} V_{\varepsilon}=-z g_{\varepsilon},\left.\quad V_{\varepsilon}(x, t)\right|_{x \in \partial D}=0, V_{\varepsilon}(x, T)=0 .
$$

Из формулы Ито (§ II.10 [8]) имеем аналогично (5.3)

$$
\begin{aligned}
V_{\varepsilon}(a, 0) & =\mathbb{E} \int_{0}^{\tau_{D} \wedge T} g_{\varepsilon}(y(t), t) \exp \left(z \int_{0}^{t} g_{\varepsilon}(y(r), r) d r\right) d t \\
& =\mathbb{E} \exp \left(z \int_{0}^{\tau_{D} \wedge T} g_{\varepsilon}(y(t), t) d t\right)-1
\end{aligned}
$$

Значит,

$$
\phi_{\varepsilon}(z)=\left(V_{\varepsilon}(\cdot, 0), \rho\right)_{H^{0}}+1=\mathbf{E} \exp \left\{z l_{\varepsilon}(T)\right\} .
$$

Отсюда получаем утверждение б) теоремы 5.1.

Докажем утверждение а). По теореме 3.1 имеем $V_{\varepsilon} \rightarrow V$ в $Y^{1}$ равномерно по $z \in \mathbf{C}:|z| \leqslant 1$. Введем функцию $\phi(z)=(V(\cdot, 0), \rho)_{H^{\circ}}+1$. По доказанному $\phi_{\varepsilon}(z) \rightarrow \phi(z)$ равномерно по $z \in \mathbf{C}:|z| \leqslant 1$. По теореме Леви это означает, что $\phi(z)$ - характеристическая функция некоторой случайной величины $\hat{\mathbf{t}}(T)$, к которой величины $l_{\varepsilon}(T)$ сходятся по распределению. Теорема 5.1 доказана.

Теорема 5.3. ІІусть $g \in X^{-1}, V=L(0) g$, погдa

$$
(V(\cdot, 0), \rho)_{H^{0}}=\mathbf{E} \hat{\mathbf{t}}(T) .
$$

Док аз а тельст во. Имеем $\mathbf{E} l_{\varepsilon}=\left(\rho, V_{\varepsilon}(\cdot, 0)\right)_{H^{0}}$, где $V_{\varepsilon}=$ $L(0) g_{\varepsilon}, g_{\varepsilon}(x, t)=\varepsilon^{-1}$ Ind $\{x \in \Gamma(t, \varepsilon)\}$. По теореме 5.1 $\mathbf{E} l_{\varepsilon}(T) \longrightarrow \mathbf{E} \hat{\mathbf{t}}(T)$ при $\varepsilon \rightarrow+0$, по теореме $3.1 V_{\varepsilon} \rightarrow V$ в $Y^{1}$. Отсюда получаем требуемое.

3 а м е ч а н и е 5.2. Для немарковских процессов Ито $y(t)$ в предположениях [9] (т.е. когда $\beta d w(t)=\widetilde{\beta} d \widetilde{w}(t)+\widehat{\beta} d \widehat{w}(t)$, где $\widetilde{\beta}, \widehat{\beta}-$ случайные неупреждающие по отношению к $\widehat{w}(t)$ функции, $\widetilde{\beta} \widetilde{\beta}^{T} \geqslant \delta I>0$ ) можно получить аналоги теорем 5.1-5.3 при помощи подхода [9]-[10].

\section{6. Явная формула для локального времени и сильная схо-} димость. Пусть выполнены предположения п. 5. Сохраняем все обозначения п. 5 (в частности, для $\left.g, l_{\varepsilon}(T), g_{\varepsilon}\right)$.

Пусть ниже $\beta_{j}, j=1, \ldots, n,-$ столбпы матрицы $\beta$ в $(1.1), \mathscr{F}_{t}-$ поток $\sigma$-алгебр событий, порожденных $\{a, w(s), s \leqslant t\}$. ПІусть $\mathscr{M}(g)$ : $X^{-1} \longrightarrow H^{0}-$ оператор, сопоставляюший функции $\varphi \in X^{-1}$ значение $\left.V(x, t)\right|_{t=0}=\mathscr{M}(g) \varphi$.

Теорема 6.1. Пусть начальный вектор а имеет плотность распределения $\rho \in L_{\infty}(D), g \in X^{-1}$, матрича $\beta(x, t)$ непрерывна. Пусть 
$V=L(0) g . \quad$ Toадa $V \in Y^{1} u \mathbf{E}\left|l_{\varepsilon}(T) \longrightarrow \hat{\mathrm{t}}(T)\right|^{2} \longrightarrow 0 n p u \varepsilon \rightarrow 0$, гдe случайная величина

$$
\hat{\mathbf{t}}(T)=V(a, 0)+\sum_{j=1}^{n} \int_{0}^{\tau_{D} \wedge T} \frac{\partial V}{\partial x}(y(t), t) \beta_{j}(y(t), t) d w_{j}(t) .
$$

Здесь под $\partial V / \partial x$ понимаем измеримую по Борелю функцию, являюиуюся представителем класса эквивалентности $\partial V / \partial x \in L_{2}(Q)$. Функции

$$
\xi_{j}(t)=\frac{\partial V}{\partial x}(y(t), t) \beta_{j}(y(t), t)
$$

таковы, ито $\mathbf{E} \int_{0}^{T}\left|\xi_{j}(t)\right|^{2} d t<+\infty \quad(\forall j)$ u для некоторой последовательности прогрессивно измеримых относительно потока $\mathscr{F}_{t}$ случайных функций времени $\xi_{\varepsilon, j}(t), \varepsilon=\varepsilon_{k} \rightarrow 0$, выполнено

$$
\mathrm{E} \int_{0}^{T}\left|\xi_{j, \varepsilon}(t)-\xi_{j}(t)\right|^{2} d t \longrightarrow 0, \quad \sup _{\varepsilon} \mathrm{E} \int_{0}^{T}\left|\xi_{j, \varepsilon}(t)\right|^{2} d t<+\infty \quad(\forall j) .
$$

Следствие 6.1. Пусть $p(x, t)$ - плотность распределения прочесса $y(t)$, обрываюшегося на $\partial D$ (т.е. $\left.p=\mathscr{M}(0)^{*} \rho\right)$. Тогда в предположениях и обозначениях теоремы 6.1 имеем

$$
\mathbf{E} \hat{\mathbf{t}}(T)^{2}=\int_{D}|V(x, 0)|^{2} \rho(x) d x+\sum_{j=1}^{n} \int_{Q}\left|\frac{\partial V}{\partial x}(x, t) \beta_{j}(x, t)\right|^{2} p(x, t) d x d t
$$

Лемма 6.1. Пусть в предположениях теоремы $6.1 V_{\varepsilon}=L(0) g_{\varepsilon}$,

$$
\xi_{j, \varepsilon}(t)=\frac{\partial V_{\varepsilon}}{\partial x}(y(t), t) \beta_{j}(y(t), t)
$$

Tогдa

$$
\ell_{\varepsilon}(T)=V_{\varepsilon}(a, 0)+\sum_{j=1}^{n} \int_{0}^{\tau_{D} \wedge T} \xi_{j, \varepsilon}(t) d w_{j}(t)
$$

$u \mathbf{E}\left|l_{\varepsilon}(T)-\hat{\mathbf{t}}(T)\right|^{2} \longrightarrow 0 n p u \varepsilon \rightarrow 0$.

Д оказа тель ст в о л е м м 6.1. Пусть $p-$ то же, что в следствии 6.1. Заметим, что $p \in L_{\infty}(Q)$. Обозначим

$$
\ell_{\varepsilon}^{x, 0}(T)=\int_{0}^{\tau_{D} \wedge T} g_{\varepsilon}\left(y^{x, 0}(t), t\right) d t
$$

Для $V_{\varepsilon}=L(0) g_{\varepsilon}$ имеем $g_{\varepsilon}=-\partial V_{\varepsilon} / \partial t-A V_{\varepsilon}$. По теореме $9.1 \S$ IV.9 [5] $V_{\varepsilon} \in W_{q}^{2,1}(Q)(\forall q>1)$. Для $x \in D$ из формулы Ито (§ II.10 [8]) имеем

$$
\begin{aligned}
-V_{\varepsilon}(a, 0) & =V_{\varepsilon}\left(y\left(\tau_{D} \wedge T\right), \tau_{D} \wedge T\right)-V_{\varepsilon}(a, 0) \\
& =-\int_{0}^{\tau_{D} \wedge T} g_{\varepsilon}(y(t), t) d t+\sum_{j=1}^{n} \int_{0}^{\tau_{D} \wedge T} \xi_{j}(y(t), t) d w_{j}(t) .
\end{aligned}
$$


Следовательно,

$$
\ell_{\varepsilon}(T)=V_{\varepsilon}(a, 0)+\sum_{j=1}^{n} \int_{0}^{\tau_{D} \wedge T} \frac{\partial V_{\varepsilon}}{\partial x}(y(t), t) \beta_{j}(y(t), t) d w_{j}(t)
$$

Отсюда получаем (6.2). Функция $\partial V_{\varepsilon}(x, t) / \partial x$ непрерывна, отсюда следует, что функции $\xi_{\varepsilon, j}(t)$ прогрессивно измеримы относительно потока $\mathscr{F}_{t}$.

Обозначим $W_{\varepsilon}=V_{\varepsilon}-V$. Имеем

$$
\begin{aligned}
& \mathbf{E} \int_{0}^{\tau_{D} \wedge T}\left|\xi_{j, \varepsilon}(t)-\xi_{j}(t)\right|^{2} d t \leqslant c \mathbf{E} \int_{0}^{\tau_{D} \wedge T}\left|\frac{\partial W_{\varepsilon}}{\partial x}(y(t), t)\right|^{2} d t \\
& \quad \leqslant c \int_{Q}\left|\frac{\partial W_{\varepsilon}}{\partial x}(x, t)\right|^{2} p(x, t) d x d t \leqslant c\|p\|_{L_{\infty}(Q)}\left\|W_{\varepsilon}\right\|_{Y^{1}} \longrightarrow 0 \\
& \mathbf{E}\left|V_{\varepsilon}(a, 0)-V(a, 0)\right|^{2} \leqslant c \int_{D}\left\|W_{\varepsilon}(x, 0)\right\|^{2} \rho(x) d x \\
& \quad \leqslant c\|\rho\|_{L_{\infty}(D)}\left\|W_{\varepsilon}\right\|_{Y^{1}} \longrightarrow 0
\end{aligned}
$$

по теореме 3.1 ( $c>0$ - некоторая константа). Лемма 6.1 доказана.

Д ок а з а т л ь с т в т т о р е м 6.1. прямо следует из леммы 6.1 и из (6.2)-(6.4).

В случае стационарных коэффициентов выведем аналог формулы Танаки (см. [1], [2], [11], [12]).

Tеорема 6.2. Пусть $\rho \in L_{\infty}(D), \beta(x, t) \equiv \beta(x), f(x, t) \equiv f(x)$, $\Gamma(t) \equiv \Gamma$ не зависят от времени, функция $\beta(x)$ непрерывна. ПІусть $F(x)$ - решение задачи $\mathscr{A} F=g,\left.F\right|_{\text {дD }}=0$ в классе $H^{1}$. Тогда $F \in C(D)$ $u$ (n.н.)

$$
\hat{\mathbf{t}}(T)=F\left(y\left(\tau_{D} \wedge T\right)\right)-F(a)-\sum_{j=1}^{n} \int_{0}^{\tau_{D} \wedge T} \frac{\partial F}{\partial x}(y(t)) \beta_{j}(y(t)) d w_{j}(t) .
$$

Здесь под $\partial F / \partial x$ понимаем измеримую по Борелю функцию, являющуюся представителем класса эквивалентности $\partial F / \partial x \in L_{2}(D)$. Функиии

$$
\widehat{\xi}_{j}(t)=\frac{\partial F}{\partial x}(y(t)) \beta_{j}(y(t))
$$

таковьі, чта $\mathbf{E} \int_{0}^{T}\left|\widehat{\xi}_{j}(t)\right|^{2} d t<+\infty(\forall j) u \mathbf{E} \int_{0}^{T}\left|\widehat{\xi}_{j, \varepsilon}(t)-\widehat{\xi}_{j}(t)\right|^{2} d t \longrightarrow 0$ для некоторой последовательности функций $\widehat{\xi}_{j, \varepsilon}(t), \varepsilon=\varepsilon_{k} \rightarrow 0$, прогрессивно измеримых по отношению $\kappa$ потоку $\mathscr{F}_{t}$ u таких, что

$$
\sup _{\varepsilon} \mathbf{E} \int_{0}^{T}\left|\widehat{\xi}_{j, \varepsilon}(t)\right|^{2} d t<+\infty \quad(\forall j) .
$$


Д оказ ат е льст в о. Пусть $F_{\varepsilon}-$ решение задачи $\mathscr{A} F_{\varepsilon}=g_{\varepsilon}$, $\left.F_{\varepsilon}\right|_{\partial D}=0$ в классе $H^{1}$. Пля $g$ выполнено (4.1), (4.2). По теореме III.14.1 $[6], F \in C(D), F_{\varepsilon} \in C(D)$ и

$$
\left\|F_{\varepsilon}-F\right\|_{C(D)} \longrightarrow 0, \quad\left\|F_{\varepsilon}-F\right\|_{H^{1}} \longrightarrow 0
$$

Пусть $V_{\varepsilon}=L(0) g, U_{\varepsilon}=V_{\varepsilon}+F_{\varepsilon}$. Имеем

$$
\frac{\partial U_{\varepsilon}}{\partial t}(x, t)+\mathscr{A} U_{\varepsilon}(x, t)=0,\left.\quad U_{\varepsilon}(x, t)\right|_{x \in \partial D}=0, U_{\varepsilon}(T, x)=F(x) .
$$

Обозначим

$$
\widehat{\xi}_{j, \varepsilon}(t)=\frac{\partial V_{\varepsilon}}{\partial x}(y(t), t) \beta_{j}(y(t)), \quad \widehat{\xi}_{j, \varepsilon}(t)=\frac{\partial F_{\varepsilon}}{\partial x}(y(t)) \beta_{j}(y(t))
$$

По лемме 6.1

$$
\ell_{\varepsilon}(T)=U_{\varepsilon}(a, 0)-F_{\varepsilon}(a)+\sum_{j=1}^{n} \int_{0}^{\tau_{D} \wedge T} \xi_{j, \varepsilon}(t) d w_{j}(t)
$$

Имеем

$$
\begin{aligned}
\xi_{j, \varepsilon}(t) & =\frac{\partial U_{\varepsilon}}{\partial x}(y(t), t) \beta_{j}(y(t))-\widehat{\xi}_{j, \varepsilon}(t) \\
F_{\varepsilon}\left(y\left(\tau_{D} \wedge T\right)\right) & =U_{\varepsilon}\left(y\left(\tau_{D} \wedge T\right), \tau_{D} \wedge T\right)
\end{aligned}
$$

По формуле Ито § ІІ.10 [8] и (6.7), имеем

$$
\begin{aligned}
& U_{\varepsilon}\left(y\left(\tau_{D} \wedge T\right), \tau_{D} \wedge T\right)=U_{\varepsilon}(a, 0) \\
& \quad+\sum_{j=1}^{n} \int_{0}^{\tau_{D} \wedge T} \frac{\partial U_{\varepsilon}}{\partial x}(y(t), t) \beta_{j}(y(t)) d w_{j}(t) .
\end{aligned}
$$

Из (6.6)-(6.12) получаем доказательство теоремы 6.2 .

3 а м е ч а н и е 6.1. Аналог теоремы 6.2 можно получить для $D=$ $\mathbf{R}^{n}$. В этом случае (6.5) выполнено для функции $F$, являющейся решением уравнения $\mathscr{A} F=g$ в классе функций полиномиального порядка роста. Известную формулу Танаки для броуновского локального времени можно записать в виде (6.5), где $n=1, D=\mathbf{R}, y(t)=a+w(t)$, $\Gamma=\{0\}, F(x)=2 x^{+}$.

\section{7. Непрерывность локального времени.}

Теорема 7.1. Пусть выполнены предположения теоремы 6.1 и сохранены обозначения этой теоремы. Пусть имеется набор аиперповерхностей $\{\Gamma(t)\}=\left\{\Gamma_{h}(t)\right\}, \Gamma_{h}(t)=\Gamma_{0}(t)+h, h \in \Delta$, где $\Delta \subset \mathbf{R}^{n}$ есть некоторое отхрытое множество, $\Gamma_{h}(t) \subset D(\forall h), 0 \in \Delta$. Iуусть $\hat{\mathbf{t}}(T)=\hat{\mathbf{t}}(T, h)$ - coответствуюшие величины для $\Gamma(t)=\Gamma_{h}(t), h \in \Delta$. Toгдa $\mathbf{E}|\hat{\mathbf{t}}(T, h)-\hat{\mathbf{t}}(T, 0)|^{2} \longrightarrow 0$ npu $h \rightarrow 0$.

2 Теория вероятностей и ее применения, № 2 
Док аз а те льсст в о. Пусть $g(t)=g^{h}(t), G_{j}^{h}(x, t)$ - соответствуюшие функции $(4.2), V^{h}=L(0) g^{h}, W^{h}=V^{h}-V^{0}$. Имеем

$$
g^{h}(t)=\sum_{j=1}^{n} \frac{\partial G_{j}^{h}}{\partial x_{j}}(x, t) .
$$

Очевидно, $G_{j}^{h}(x, t) \equiv G_{j}^{0}(x-h, t),\left\|G_{j}^{h}-G_{j}^{0}\right\|_{L_{2}(Q)} \longrightarrow 0$ при $h \rightarrow 0, j=$ $1, \ldots, n$, тогда $\left\|g^{h}-g^{0}\right\|_{X^{-1}} \longrightarrow 0$. По теоремам $3.1,6.1$,

$$
\begin{aligned}
& \mathbf{E}|\hat{\mathbf{t}}(T, h)-\hat{\mathbf{t}}(T, 0)|^{2} \leqslant c \mathbf{E}\left|V^{h}(a, 0)-V^{0}(a, 0)\right|^{2} \\
& \quad+c \mathbf{E} \int_{0}^{\tau_{D} \wedge T}\left|\frac{\partial W^{h}}{\partial x}(y(t), t)\right|^{2} d t \\
& \leqslant c \int_{D}\left\|W^{h}(x, 0)\right\|^{2} \rho(x) d x+c \int_{Q}\left|\frac{\partial W^{h}}{\partial x}(x, t)\right|^{2} p(x, t) d x d t \\
& \leqslant c\|p\|_{L_{\infty}(Q)}\left\|W^{h}\right\|_{Y^{1}} \leqslant c\|p\|_{L_{\infty}(Q)}\left\|g^{h}-g^{0}\right\|_{X^{-1}} \longrightarrow 0
\end{aligned}
$$

для некоторой константы $c>0$. Теорема 7.1 доказана.

Теорема 7.2. Пусть сохраняются предположения и обозначения теоремы 6.1. Пусть $T_{0} \in[0, T], T_{1} \in[0, T]$. Тогда $\mathbf{E}\left|\hat{\mathbf{t}}\left(T_{1}\right)-\hat{\mathbf{t}}\left(T_{0}\right)\right|^{2} \longrightarrow 0$ npu $T_{1} \rightarrow T_{0}$.

Док а за т ель с т во. Пусть $\varphi_{0} \in X^{-1}, \varphi_{1} \in X^{-1}, \varphi_{i}(t)=g(t)$ для $t \leqslant T_{i}, \varphi_{i}(t)=0$ для $t>T_{i}, i=0,1$. Пусть $V_{i}=L(0) \varphi_{i}, W=$ $V_{1}-V_{0}$. Очевидно, $\left\|\varphi_{1}-\varphi_{0}\right\|_{X^{-1}} \longrightarrow 0$. По теореме $3.1,\|W\|_{Y^{1}} \rightarrow 0$. Далее доказательство теоремы 7.2 аналогично доказательству теоремы 7.1.

Теорема 7.3. Пусть выполнены предположения теоремы 6.2. Тогда существует вариант $\hat{\mathbf{t}}(T)$, n.н. непрерывный по $T$.

Доказательство является прямым следствием (6.5).

8. Вырождение диффузии. В работах автора [7], [13] изучен ряд вопросов, связанных с предельными свойствами процессов при вырождении диффузии (включая распределения функционалов от негладких функций [13] и от моментов первого выхода из области [7]). Здесь подход [7], [13] применен к предельным свойствам локального времени в случае стремящейся к нулю диффузии. Установлено, что локальное время случайных траекторий в-некотором смысле стремится к локальному времени предельной глацкой функции после усреднения по начальным значениям (хотя траектории все время остаюося стохастически негладкими и по винеровскому процессу усреднение не ведется).

Рассмотрим набор коэффициентов диффузии

$$
\beta=\beta_{\delta}, \quad \beta_{\delta}(x, t) \equiv \sqrt{\delta} B(x, t),
$$

где число $\delta \geqslant 0, B(x, t): \mathbf{R}^{n} \times \mathbf{R} \longrightarrow \mathbf{R}^{n \times n}-$ такая матричная функция, 
что $B(x, t) B(x, t)^{\mathrm{T}} \geqslant I$. Полагаем, что при $\delta>0$ выполнены все предположениях п. $1, D=\mathbf{R}^{n}$, производные $\partial^{m} f(x, t) / \partial x^{m}, \partial^{m} \beta(x, t) / \partial x^{m}$ ограничены, $m=1,2,3,4$, случайный начальный вектор $a$ имеет плотность распределения $\rho \in L_{\infty}\left(\mathbf{R}^{n}\right), B(x, t)-$ непрерывная функция. Полагаем, что гиперповерхность $\Gamma(t) \subset \mathbf{R}^{n}$ зависит от времени и для нее выполнены предположения теоремы $5.1, g(t)$ - тот же элемент $\mathscr{W}^{*}$, что в теореме $4.1, g \in X^{-1}$.

Для $\delta>0$ через $\hat{\mathbf{t}}_{\delta}(T)$ обозначаем величину, введенную в теореме 6.1 ; используем обозначения $y_{\delta}(t), L_{\delta}(0), \mathscr{M}_{\delta}(0)$ для соответствующих процессов $y(t)$ и введенных в п. 3 , п. 6 операторов $L(0), \mathscr{M}(0)$.

В частности, оператор $\mathscr{M}_{\delta}(0): X^{0} \longrightarrow H^{0}$ сопоставляет функции $\varphi$ функцию $V(\cdot, 0) \in H^{1}$, где $V=L_{\delta}(0) \varphi, \delta>0$. Переходя от параболических уравнений к уравнениям первого порядка, вводим аналогичные операторы для $\delta=0$. По теореме 4.2.1 [14] (относяшейся к более общим уравнениям) оператор $\mathscr{M}_{0}^{*}(0): H^{1} \longrightarrow X^{1}$ непрерывен. Определим оператор $\mathscr{M}_{0}(0): X^{-1} \longrightarrow H^{-1}$ как сопряженный к нему.

Аналогично, по теореме 4.2.1 [14], операторы $L_{\delta}^{*}(0): X^{k} \longrightarrow X^{k}$, $\mathscr{M}_{\delta}^{*}(0): H^{k} \longrightarrow X^{k}$ непрерывны и их нормы ограничены по $\delta \in[0,1]$, $k=0,1,2$. Для сопряженных операторов отсюда следует

Предложение 8.1. Oператоры $L_{\delta}(0): X^{-k} \longrightarrow X^{-k}, \mathscr{M}_{\delta}(0)$ : $X^{-k} \longrightarrow H^{-k}$ непрерывны и их нормы ограничены по $\delta \in[0,1], k=0,1,2$.

Пусть, как и выше, $g_{\varepsilon}(x, t)=\operatorname{Ind}\{x \in \Gamma(t, \varepsilon)\}$. Обозначаем

$$
u_{\delta, \varepsilon}=\mathscr{M}_{\delta}(0) g_{\varepsilon}, \quad u_{\delta, 0}=\mathscr{M}_{\delta}(0) g .
$$

Лемма 8.1. а) Нормы $\left\|u_{\delta, \varepsilon}\right\|_{H^{-1}}$ ограничены равномерно по $\varepsilon \in$ $(0,1], \delta \in[0,1]$.

б) Дая $V_{\delta, \varepsilon}=L_{\delta}(0) g_{\varepsilon}, V_{\delta}=L_{\delta}(0) g . \quad$ нормы $\left\|V_{\delta, \varepsilon}(\cdot, t)\right\|_{H^{-1}}$, $\left\|V_{\delta}(\cdot, t)\right\|_{H^{-1}}$ ограничены равномерно по $\left.t \in[0, T], \varepsilon \in(0,1], \delta \in[0,1]\right)$.

Д ок а за т е л ь с т в о. Утверждение а) и утверждение $б$ ) для фиксированного $t=0$ следует сразу же из предложения 8.1, утверждение б) для всех $t$ получаем заменой начального времени.

Обозначим $y_{0}^{x, 0}(t)$ решение уравнения (1.1)-(1.2) с $\beta \equiv 0$ и начальным условием $y(0)=x, x \in \mathbf{R}^{n}$.

Предложение 8.2. Имеет место равенство в $H^{0}=L_{2}\left(\mathbf{R}^{n}\right)$ :

$$
\frac{1}{\varepsilon} \int_{0}^{T} \operatorname{Ind}\left\{y_{0}^{x, 0}(t) \in \Gamma(\varepsilon, t)\right\} d t=u_{0, \varepsilon}(x)=V_{0, \varepsilon}(x, 0) .
$$

Кроме того, для $\delta>0$ выполнено

$$
\frac{1}{\varepsilon} \mathbf{E} \int_{0}^{T} \operatorname{Ind}\left\{y_{\delta}(t) \in \Gamma(\varepsilon, t)\right\} d t=\left(\rho, u_{\delta, \varepsilon}\right)_{H^{0}}
$$

(Здесь первое равенство следует из теоремы 1.1 [13], второе - из теоремы 5.3.) 
Теорема 8.1. $u_{\delta, \varepsilon} \rightarrow u_{0,0}$ слабо в $H^{-1}$ при $\delta \rightarrow 0, \varepsilon \rightarrow 0$.

Из предложения 8.1 и теоремы 8.1 видно, что естественным является следуюшее определение.

$\mathrm{O}$ п р е д е л е н и е 8.1. Локальным временем пребывания на $\Gamma$ до момента $T$ для набора траекторий $\left\{y_{0}^{x, 0}(t)\right\}_{x \in \mathbf{R}^{n}}$ называем $u_{0,0} \in H^{-1}$.

Отметим, что если выполнено $u_{0,0} \in H^{0}$, то $u_{0,0}=u_{0,0}(x)$ является измеримой по Лебегу функцией от $x$. Приведем пример, в котором $u_{0,0} \in H^{0}$, локальное время задано для любого $x=y_{0}^{x, 0}(0)$ и является не обобщенной, а ограниченной измеримой функцией от $x$.

П р и м е р 8.1. Пусть $n=1, D=\mathbf{R}, \Gamma(t) \equiv\{0\}$. ІІолагаем, что функции $f(x, t), B(x, t)$ измеримы и ограничены вместе со всеми производными по $x,|B(x, t)| \geqslant 1$, число $\delta \geqslant 0$, функция $f(\cdot, t):[0, T] \longrightarrow C^{1}(\mathbf{R})$ кусочно-непрерывна, $|f(0, t)| \geqslant c_{1}(\forall t)$, где $c_{1}>0$ - некоторая константа. Тогда $u_{0,0}(x)=\sum_{k=1}^{N} s\left(\theta_{k}\right)\left|f\left(0, \theta_{k}\right)\right|^{-1}$, где $N$ - число посещений нуля процессом $y_{0}^{x, 0}(t)$ при $t \in[0, T], \theta_{k}-$ моменты времени посещения, $k=1, \ldots, N, s(t)=1$ при $t \in(0, T), s(0)=s(T)=\frac{1}{2}$.

До к аз ат тльс т в о те о ре мы 8.1.

Лемма 8.2. Последовательность $u_{\delta, 0}$ слабо сходится $\boldsymbol{\kappa} u_{0,0}$ при $\delta \rightarrow 0$ в пространстве $\mathrm{H}^{-1}$.

Д о к а $з$ а т л л ь с т в о. Введем множество $B^{+}=\left\{\xi \in H^{1}: \xi(x) \geqslant 0\right.$, $\left.\|\xi\|_{L_{1}\left(\mathbf{R}^{n}\right)}=1\right\}$. Для $\xi \in B^{+}$обозначим $p_{\delta}=\mathscr{M}_{\delta}^{*}(0) \xi$. Заметим, что функция $p_{\delta}(x, t)$ является плотностью распределения решения $y(t)=y_{\delta}(t)$ уравнения $(1.1),(1.2)$ при условии, что вектор $y(0)$ имеет плотность распределения $\xi(x)$, с учетом (8.1). Известно, что $\mathbf{E} \sup _{t}\left|y_{\delta}(t)-y_{0}(t)\right|^{2} \longrightarrow 0$. Отсюда получаем, что $p_{\delta} \rightarrow p_{0}$ слабо в $X^{0}$ при $\delta \rightarrow 0$. По предложению 8.1, $\left\|p_{\delta}\right\|_{X^{1}} \leqslant$ const $(\forall \delta \in[0,1])$. Значит,

$$
p_{\delta} \rightarrow p_{0} \quad \text { слабо в } X^{1} \text { при } \delta \rightarrow 0 .
$$

Отсюда следует, что для любого $\xi \in B^{+}$выполнено

$$
\left(u_{\delta, 0}-u_{0,0}, \xi\right)_{H^{0}}=\left(g, p_{\delta}-p_{0}\right)_{X^{0}} \longrightarrow 0 .
$$

Јинейная оболочка $B^{+}$плотно в $H^{1}$; отсюда, из леммы 8.1 и соотношения (8.4) получаем лемму 8.2 .

Продолжим доказательство теоремы 8.1. В силу леммы 8.1 достаточно доказать, что $u_{\delta, \varepsilon} \rightarrow u_{0,0}$ слабо в $H^{-2}$ при $\delta \rightarrow 0, \varepsilon \rightarrow 0$. Пусть $\xi \in H^{2}, u_{\delta, 1}, u_{\delta, 0}$ заданы (8.2). Имеем

$$
\left(u_{\delta, \varepsilon}-u_{0,0}, \xi\right)_{H^{0}}=R_{1}(\delta)+R_{2}(\delta, \varepsilon),
$$

где

$$
R_{1}(\delta)=\left(u_{\delta, 0}-u_{0,0}, \xi\right)_{H^{\circ}}, \quad R_{2}(\delta, \varepsilon)=\left(u_{\delta, \varepsilon}-u_{\delta, 0}, \xi\right)_{H^{\circ}}
$$


Имеем $R_{1}(\delta) \rightarrow 0$ при $\delta \rightarrow 0$ по лемме 8.1 ,

$$
\begin{aligned}
R_{2}(\delta, \varepsilon) & =\left(\mathscr{M}_{\delta}(0)\left(g_{\varepsilon}-g\right), \xi\right)_{H^{0}}=\left(g_{\varepsilon}-g, \mathscr{M}_{\delta}^{*}(0) \xi\right)_{H^{0}} \\
& \leqslant\left\|g_{\varepsilon}-g\right\|_{X^{-1}}\left\|\mathscr{M}_{\delta}^{*}(0) \xi\right\|_{X^{1}} \leqslant \text { const }\left\|g_{\varepsilon}-g\right\|_{X^{-1}}\|\xi\|_{H^{1}}
\end{aligned}
$$

Теорема 8.1 доказана.

Пусть $\mathscr{F}_{a}, \mathscr{F}_{W}$ суть $\sigma$-алгебры событий, порожденные соответственно начальным вектором $a$ в (1.1) и винеровским процессом $w(s), s \in$ $[0, T] ;(\Omega, \mathscr{F}, \mathbf{P})$ - исходное вероятностное пространство, $\Omega=\{\omega\}$.

Пусть $V_{\delta}=L_{\delta}(0) g, \beta_{\delta}^{(j)}-$ столбцы матрицы $\beta_{\delta}$. По теореме 6.1 имеем

$$
\hat{\mathbf{t}}_{\delta}(T)=V_{\delta}(a, 0)+\sum_{j=1}^{n} \int_{0}^{T} \frac{\partial V_{\delta}}{\partial x}\left(y_{\delta}(t), t\right) \beta_{\delta}^{(j)}\left(y_{\delta}(t), t\right) d w_{j}(t) .
$$

Обозначим

$$
\eta_{\delta}=\hat{\mathbf{t}}_{\delta}(T)-\mathbf{E}\left\{\hat{\mathbf{t}}_{\delta}(T) \mid \mathscr{F}_{a}\right\}, \quad \bar{\eta}_{\delta}=\mathbf{E}\left\{\eta_{\delta} \mid \mathscr{F}_{W}\right\} .
$$

Имеем из (8.5)

$$
\eta_{\delta}=\sum_{j=1}^{n} \int_{0}^{T} \frac{\partial V_{\delta}}{\partial x}\left(y_{\delta}(t), t\right) \beta_{\delta}^{(j)}\left(y_{\delta}(t), t\right) d w_{j}(t) .
$$

Теорема 8.2. а) Для любого $\rho \in H^{1}$ выполнено

$$
\mathbf{E} \hat{\mathbf{t}}_{\delta}(T) \longrightarrow\left(u_{0,0}, \rho\right)_{H^{0}} \quad \text { npu } \delta \rightarrow 0 .
$$

б) ПІусть $\rho \in H^{2}$. Тогдa

$$
\mathbf{E}\left|\bar{\eta}_{\delta}\right|^{2} \leqslant \delta \cdot \text { const }
$$

Д о к а з а т е л ь с т в о. Соотношение (8.8) следует из (8.5) и леммы 8.2. Докажем (8.9). Пусть $p(x, t, \omega)-$ условная относительно $\mathscr{F}_{W}$ плотность распределения процесса $y_{\delta}(t)$, т.е. для любой области $G \subset \mathbf{R}^{n}$

$$
\int_{G} p(x, t, \omega) d x=\mathbf{P}\left\{y_{\delta}(t) \in G\right\} .
$$

Напомним, что $\mathscr{C}^{k}=C\left([0, T] \longrightarrow H^{k}\right)$.

Предложение 8.3. Пусть вектор $a=y_{\delta}(0)$ не зависит от $w(t)$ $u$ имеет плотность распределения $\rho \in H^{0}$. Тогда существует условная относительно $\mathscr{F}_{W}$ плотность распределения прочесса $y_{\delta}(t): p=$ $p(x, t, \omega) \in L^{2}\left(\Omega, \mathscr{F}, \mathbf{P}, \mathscr{C}^{0}\right)$. Ecsu $\rho \in H^{k}$, mo $p(x, t, \omega) \in L^{2}\left(\Omega \mathscr{F}, \mathbf{P}, \mathscr{C}^{k}\right), u$

$$
\mathbf{E}\|p(\cdot, \omega)\|_{\mathscr{C}^{2}}^{2} \leqslant \text { const } \mathbf{E}\|\rho\|_{H^{k}}^{2}, \quad k=0,1,2 .
$$


Это предложение следует из теоремы 2.2 [10]; последняя оценка выполнена по теореме 4.2.1 [14] в силу свойств параболического уравнения для $p$, выведенного в теореме 5.3.1 [14].

Продолжим доказательство теоремы 8.2. Имеем

$$
\begin{aligned}
\bar{\eta}_{\delta} & =\sum_{j=1}^{n} \int_{0}^{T} d w_{j}(t) \int_{\mathbf{R}^{n}} \frac{\partial V_{\delta}}{\partial x}(x, t) \beta_{\delta}^{(j)}(x, t) p(x, t, \omega) d x \\
\mathbf{E}\left|\bar{\eta}_{\delta}\right|^{2} & =\mathbf{E} \sum_{j=1}^{n} \int_{0}^{T} d t \int_{\mathbf{R}^{n}}\left|\frac{\partial V_{\delta}}{\partial x}(x, t) \beta_{\delta}^{(j)}(x, t) p(x, t, \omega)\right|^{2} d x \\
& \leqslant \operatorname{const} \mathbf{E} \sum_{j=1}^{n} \int_{0}^{T}\left\|V_{\delta}(\cdot, t)\right\|_{H^{-1}}^{2}\left\|\beta_{\delta}^{(j)}(x, t) p(x, t, \omega)\right\|_{H^{2}}^{2} d t \\
& \leqslant \delta \text { const } \mathbf{E}\|p(\cdot, \omega)\|_{X^{2}}^{2} \leqslant \delta \text { const } \mathbf{E}\|\rho\|_{H^{2}}^{2} .
\end{aligned}
$$

Предпоследнее неравенство выполнено в силу (8.1) и леммы 8.1a). Теорема 8.2 доказана.

3 а м е ч а н и е 8.1. Пусть $\rho \in W_{2}^{k}\left(\mathbf{R}^{n}\right), k>n / 2+2, \delta>0$ фиксировано, производные $\partial^{m} f / \partial x^{m}, \partial^{m} \beta / \partial x^{m}$ ограничены, $m=1, \ldots, k+2$. Применяя подход, использованный выше для теоремы 8.2, можно доказать, что существует предел при $\varepsilon \rightarrow+0$ в пространстве $L^{2}(\Omega, \mathscr{F}, \mathbf{P})$ у величин $\bar{l}_{\varepsilon}(x, t)$, где $x \in \mathbf{R}^{n}, t>0$,

$$
\bar{l}_{\varepsilon}(x, t)=\mathbf{E}\left\{l_{\varepsilon}(x, t) \mid \mathscr{F}_{W}\right\}, \quad l_{\varepsilon}(x, t)=\frac{1}{\varepsilon^{n}} \int_{0}^{t} \operatorname{Ind}\{|y(r)-x|<\varepsilon\} d r .
$$

(Заметим, что при $n>1$ не существует предел величин $l_{\varepsilon}(x, t)$ без условного усреднения, поскольку, как известно, локальное время пребывания многомерного процесса в точке не сушествует.)

В заключение отметим, что на основе теоремы 3.1 в [15], [16] решались задачи управления с функционалами, зависящими от локального времени. Кроме того, теоремы 4.1, 5.1 позволяют интерпретировать задачи, решаемые в [17], как задачи управления локальным временем.

\section{СПИСОК ЛИТЕРАТУРЫ}

1. Geman D., Horovitz J. Occupation density. - Ann. Probab., 1980, v. 8, № 1, p. 1-67.

2. Бородия $A$. H. Броуновское локальное время. - Успехи матем. наук, 1989, т. 44, B. 2 , c. $7-48$.

3. Бородия A. Н., Нбрагимов И. А. Предельные теоремы для функционалов от случайных блужданий. - Тр. Матем. ин-та им. В. А. Стеклова. 1994. Т. 195, $285 \mathrm{c}$.

4. Bass R. F. Occupation times of $d$-dimensional semimartingales. - Progr. Probab. Statist., 1983, v. 5, p. 51-96 (Seminar on Stochastic Processes, 1982). 
5. Ладыженская O. А., Солоняиков $B$. ., Уральчева $H$. Н. Линейные и квазилинейные уравнения параболического типа. М., 1967.

6. Ладыженская O. А., Уральчева $H$. Н. Линейные и қвазилинейные уравнения эллиптического типа. М., 1968.

7. Докучаев $Н$. Г. Интегральные оценки для решений обыкновенных дифференциальных уравнений при обрыве на границе области. - Дифференц. уравнения, 1992, т. 28, № 11, c. 1890-1900.

8. Крылов Н. В. Управляемые процессы диффузионного типа. М.: Наука, 1977, $399 \mathrm{c}$.

9. Докучаев $H . Г$. Краевые задачи для функционалов от процессов Ито. Теория вероятн. и ее примен., 1991, т. 36, в. 3, с. 464-481.

10. Докучаев Н. Г. Распределения процессов Ито: оценки для плотности и для условных ожиданий интегральных функционалов. - Теория вероятн. и ее примен., 1994 , т. 39, в. 4, с. 825-833.

11. Ватанабэ фузионные процессы. М.: Наука, 1986.

12. Насыров Ф. С. О локальных временах для функций и случайных процессов. Ч. І, II. - Теория вероятн. и ее примен., 1995 , т. 40, в. 4, с. $798-812 ; 1996$, т. 41 , в. 2, c. 284-299.

13. Докучаев Н. Г. Интегральные оценки для решений обыкновенных дифференциальных уравнений и их применения к негладким задачам оптимального управления. - Дифференц. уравнения, 1991, т. 27, № 10, с. 1671-1691.

14. Розовский Б. Л. Эволющионные стохастические системы. Линейная теория и приложения к статистике случайных процессов. М.: Наука, 1983, 208 с.

15. Докучаев Н. Г. Стохастические задачи управления для функционалов от локального времени. - Вестн. С.-Петербург. ун-та, сер. 1, 1997, в. 2, № 8, с. 112-114.

16. Докучаев Н. Г. Уравнения для распределений локального времени пребывания диффузионного процесса на поверхности и задачи управления. - Записки научн. семин. ПОМИ, 1997 , т. 244 , с. $96-118$.

17. Докучаев $Н$. Г. Параболические уравнения без граничного условия Коши и задачи управления диффузионными процессами. Ч. 2. -- Дифференц. уравнения, 1995 , т. 31 , № 8, c. $1409-1418$.

Поступила в редакцию 